\title{
Trade-Off Between Cost and Time to the Project to Assist in Decision-Making Using Multi-Objective Genetic Algorithm (MOGA)
}

\author{
Sarmad Nasser Mohammed and Ahmed Abdulrasool Alkhafaji \\ Department of Mechanical Engineering, College of Engineering, \\ University of Baghdad, Baghdad, Iraq
}

\begin{abstract}
The time-cost trade-off analysis is a usual problem in the project management world and it is one of the most important aspects of project planning and control. Project management focuses on completing the project in the least time and at the lowest possible cost in general. This problem represents a challenging task because the activity duration and cost have uncertainty associated with management which should be considered when performing schedule optimization. This study uses Multi-Objective Genetic Algorithm (MOGA) to trade-off between time and cost which programmed with the MATLAB 2017b based on the concept of the Pareto front and the Excel program used as an input tool for the project data. A case study was solved to show clearly how the algorithm works and how it trade-offs between time and cost. The results showed that the algorithm helps to find optimal solutions quickly and efficiently with high accuracy to clarify how it could be useful for project management by helping to take the right decision to choose the crew and the appropriate method for each activity.
\end{abstract}

Key words: MOGA, project management, time-cost trade-off, Pareto front, MATLAB, method

\section{INTRODUCTION}

Project management is the application of knowledge, skills, tools and techniques to project activities to meet project requirements (Afruzi et al., 2013) in general the project is a set of activities to achieve a certain objectives (Hooshyar et al., 2008). Scheduling of large projects containing a large number of activities constitute a major problem for the management, especially, projects which its activities contain several methods possible to execute it and of the most important problems faced by the management is the problem of trade-off between the time of the project and its total cost, the objective of typical time-cost trade-off problem is to identify the set of time-cost alternatives that will minimize the total cost of the project (Aminbakhsh and Sonmez, 2016). Time and cost are very critical objectives and are linked to each other. Decision-makers always need to take into account the trade-off between the total project cost and the project completion time. Sometimes interested in reducing the project cost, decisions may be made with the sacrifice of extending the project completion time. In other cases, decision-makers may need to adjust the schedule of the project to increase the project cost for the demand of finishing the project in time. For example, the decision to purchase more equipment or hire more workers can accelerate performing the project such that the project can be finished before the due date on the other hand the total project cost increases consequently. Therefore, it is logically desirable for decision-makers to find a schedule to complete a project with least time and lowest possible cost, to satisfy the project completion time constraint (Ke et al., 2009).

The traditional method of crashing CPM/PERT networks only considers average activity times for the calculation of the critical path, ignoring the uncertainty related to the duration of the activities. Consequently, other paths that may have a high probability of becoming critical are ignored (Sahu and Sahu, 2014). And using $\mathrm{CPM} / \mathrm{PERT}$ networks in large and complex projects is difficult and takes a long time to reach optimal solutions, especially in projects that show their activities more than two options to execute them. There are two methods to multiple-objective optimization. One is to combine the individual objective functions into a single compound function. The second method is to determine an entire Pareto optimal solution set (Konak et al., 2006). Though moving from one Pareto solution to another, there is always a certain amount of loss in one objective to attain a certain amount of increase in the other (Konak et al., 2006; Van Sickel et al., 2008). Pareto optimal solution sets are often favored to single solutions because they can be

Corresponding Author: Sarmad Nasser Mohammed, Department of Mechanical Engineering, College of Engineering, University of Baghdad, Baghdad, Iraq 
useful when taking into consideration real-life problems because the final solution of the decision-maker is always a trade-off and minimization the objectives (Konak et al., 2006). So, in this study was used multi-objectives GA, to trade-off the time and cost based on Pareto front, Pareto front is a set of solutions that are not stand out with respect to each other (Konak et al., 2006). Which explains the optimal solutions and helps to make decisions on the best set project completion time on depend on the company's physical budget. A Genetic algorithm can be defined as a research-based technique based on the principle of genetics and natural selection and is used to find optimal solutions to difficult and complex problems that may need a very long time to solve and is used as a tool for optimization. Bajpai and Kumar (2010) some researchers who have focused the problem of cost-time trade-off using artificial intelligence (Hooshyar et al., 2008): presented a Genetic algorithm to solve the problem of the Time-Cost Trade-Off (TCTP) and they used two control variables also they presented an intelligent mutation operator, then comparing the proposed algorithm with siemens algorithm. Ding et al. (2010) proposed approach to estimate the uncertainty of cost and time based on trapezoid fuzzy numbers and then applied a-cut method to determine the risk level and to make possibility of large scale computation they improved mutation and crossover methods for Multi-Objective Genetic Algorithm MOGA (Paryzad and Pour, 2013): a meta heuristic and heuristic IWO (Invasive Weed Optimization) algorithm is introduced for solving the time, cost and quality problem. They create a balance between cost, time and quality. Mazlum and Guneri (2015) discuss fuzzy PERT and fuzzy CPM in the fuzzy project management that used to improve an online internet branch and to plan the project of an online internet branch.

The aim of the research is to find the best trade-off between the time of the project and its cost, based on change the working methods of activities using multi-objective Genetic algorithm which is a tool of optimization to facilitate the decision-making processs the algorithm was programmed with the in MATLAB (2017b).

Multi-objective fitness function: Multi-objective optimization or Pareto optimization is an space of several criteria decision making that is involved with arithmetical optimization problems including more than one objective function to be improved simultaneously (Paryzad and Pour, 2013). The multi-objectives algorithm needs to be equated to each objective. Therefore, we need an equation to calculate the total time of the project which is the first objective and another equation to evaluate the cost of the project based on the times of its activities. The algorithm will trade-off between the two objectives based on the time equation and the cost equation. The values of fitness will be two dimensions which are the project time and its total cost.

The first objective in the problem of time-cost trade-off is to reduce the overall duration of the project. This objective is as in Eq. 1 which determines the longer path of network diagram:

$$
\mathrm{T}_{\mathrm{t}}=\operatorname{maximum} \sum_{\mathrm{i}=1}^{\mathrm{k}} \mathrm{t}_{\mathrm{i}}
$$

Where:

$\mathrm{i}=$ Activity index $(1,2,3,4, \ldots, \mathrm{k})$

$\mathrm{k}=$ The number of activities

$\mathrm{t}_{\mathrm{i}}=$ The duration of activity (i) on path

$\mathrm{T}_{\mathrm{t}}=$ Total duration of the project that equals to longest path on the network diagram

The second objective in the trade-off between time and cost problem is reduce the total cost of the project, the total cost of the project is calculated according to Eq. 2:

$$
C_{t}=\sum_{i=1}^{k}\left(D C_{i}+t_{i} \times I C\right)
$$

Where:

$C_{t}=$ Total Cost of all activities on network diagram

$\mathrm{DC}_{\mathrm{i}}=$ Direct Cost of activity $\mathrm{i}$

IC $=$ Indirect Cost

Based on the Pareto optimality, optimization can be set as (Paryzad and Pour, 2013; Mazlum and Guneri, 2015):

$$
\mathrm{f}=\text { Minimum( }(\mathrm{f} \text { of durations total } \mathrm{f} \text { of total } \cos \mathrm{t})
$$

where, $\mathrm{f}=$ solutions space.

\section{MATERIALS AND MEHTODS}

In this study, MOGA was used to trade-off between project time and its cost to reach the optimal solution. The algorithm deals with the principle of chromosomes and mating with each other and exchange genes, according to the fitness equations used. As illustrated in the block diagram in Fig. 1, the Excel program was used as an input tool for project activity data and their precedence relationship, the algorithm is programmed in MATLAB 2017b. The data of the project activities imported into Excel are read by MATLAB and processed and analyses the best trade-off between the total time of the project and its total cost. The operation will take place chromosomes with genes whose activities have high time 


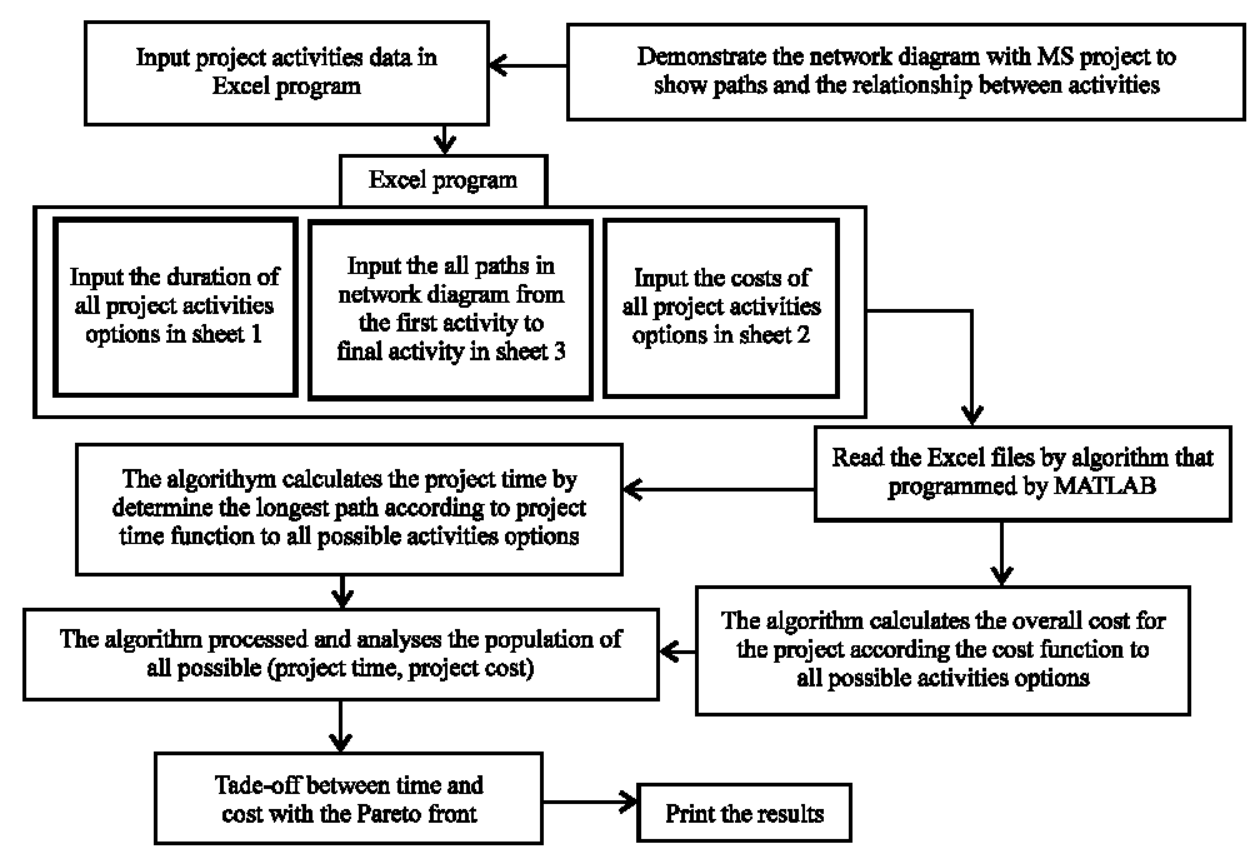

Fig. 1: The procedure of reading and processing data

values are selected first, fitness function calculates the total time of the project with Eq. 1 and its total cost is with Eq. 2. Trade-off between the two objectives are by the Pareto optimized tool where Pareto optimality is a tool within the multi-goal Genetic algorithm The time of the activities is changed by changing the methods that the activity will be performed. The process is repeated until optimal values are reached.

The trade-off between the project's time and its totalcost is graphically represented and set of optimal solutions are presented with Pareto front, so that, the decision-maker can choose the best time for the company depending on the limits budget allocated for project completion. A case study of one of the projects described in Table 1 has 14 activities from A-N shows how the MOGA works and reach several optimal solutions. In this case study, each activity has several options in its execution. For example, activity $\mathrm{C}$ (Build the wall) can be executed in method 1 (crew 1) and its estimated time is 10 weeks and at a cost of $\$ 620000$. It can be implemented in method 2 (crew 2) which takes 7 weeks at a cost of $\$ 860000$ or it can be implemented by method 3 (crew 3), Similarly to all activities. We Assume indirect cost $=\$ 200 /$ week. It is difficult for the decision-maker to make a decision about which method is chosen for each activity in order to arrive at the optimal decision for the whole project which is an optimal solution of trade-off between the time of the project and its cost.
Based on the predecessor and successor relationships of these activities, the network diagram of the example will be as shown in Fig. 2 .

The network was drawn by the MS project program. The algorithm calculates the total time and the overall cost of the project with the critical path method CPM and by taking into account the relationship between the activities of precedence described in the project's network diagram, note that the critical path changes in the network with changing work options for activities and each time the total time of the project is calculated by taking the longest path to the network which is the critical path. Our idea is to represent the project data with a chromosome. The length of the chromosome string is equal to the number of project activities that is each gene in the chromosome is an activity. And that each chromosome represents a possible solution. Figure 3 demonstrates how the project is represented by chromosomes and illustrate the process of changing genes between chromosomes.

Chromosomes represent possible solutions for example, chromosome 3 , activity A (Dig) will be performed with method 2 (Two excavator) and activity $\mathrm{E}$ will be performed with method 1(crew 1) and similarly to all chromosomes showing possible solutions to the project, this solution well improved by exchanging the activity options based on crosseovers between gens. Figure 4 illustrations a flow chart for the implementation MOGA procedure. 
Table 1: The time and cost for a case study of a project activities for each method

\begin{tabular}{|c|c|c|c|c|}
\hline Activity & Activity symbol & Job methods & Duration (weeks) & Direct cost $(\$)$ \\
\hline \multirow[t]{2}{*}{ Dig } & $\mathrm{A}$ & One excavator & 2 & 180000 \\
\hline & & Two excavator & 1 & 280000 \\
\hline \multirow[t]{2}{*}{ Lay the groundwork } & B & Crew 1 & 2 & 320000 \\
\hline & & Crew 2 & 4 & 420000 \\
\hline \multirow[t]{3}{*}{ Build the wall } & $\mathrm{C}$ & Method 1 & 10 & 620000 \\
\hline & & Method 2 & 7 & 860000 \\
\hline & & Method 3 & 5 & 1000000 \\
\hline \multirow[t]{2}{*}{ Put up the roof } & $\mathrm{D}$ & Method 1 & 6 & 260000 \\
\hline & & Method 2 & 4 & 340000 \\
\hline Set up the & $\mathrm{E}$ & Crew 1 & 4 & 410000 \\
\hline exterior water system & & Crew 2 & 3 & 570000 \\
\hline Set up the interior & $\mathrm{F}$ & Crew 1 & 5 & 180000 \\
\hline heating system & & Crew 2 & 3 & 260000 \\
\hline \multirow[t]{2}{*}{ Erect the exterior side wall } & G & Method 1 & 7 & 900000 \\
\hline & & Method 2 & 4 & 1020000 \\
\hline \multirow[t]{3}{*}{ Perform the external pigment } & $\mathrm{H}$ & Method 1 & 9 & 200000 \\
\hline & & Method 2 & 6 & 380000 \\
\hline & & Method 3 & 4 & 500000 \\
\hline \multirow[t]{3}{*}{ Perform the electrical work } & I & Crew 1 & 7 & 210000 \\
\hline & & Crew 2 & 5 & 270000 \\
\hline & & Crew 3 & 4 & 310000 \\
\hline \multirow[t]{2}{*}{ Put up the wallpaper } & $\mathrm{J}$ & Method 1 & 8 & 430000 \\
\hline & & Method 2 & 6 & 490000 \\
\hline \multirow[t]{2}{*}{ Put in the floorboards } & $\mathrm{K}$ & Method 1 & 4 & 160000 \\
\hline & & Method 2 & 3 & 200000 \\
\hline \multirow[t]{2}{*}{ Perform the interior pigment } & $\mathrm{L}$ & Method 1 & 5 & 250000 \\
\hline & & Method 2 & 3 & 350000 \\
\hline \multirow[t]{2}{*}{ Set up the outside fittings } & $\mathrm{M}$ & Crew 1 & 2 & 100000 \\
\hline & & Crew 2 & 1 & 200000 \\
\hline \multirow[t]{2}{*}{ Set up the interior fittings } & $\mathrm{N}$ & Method 1 & 6 & 330000 \\
\hline & & Method 2 & 3 & 510000 \\
\hline
\end{tabular}

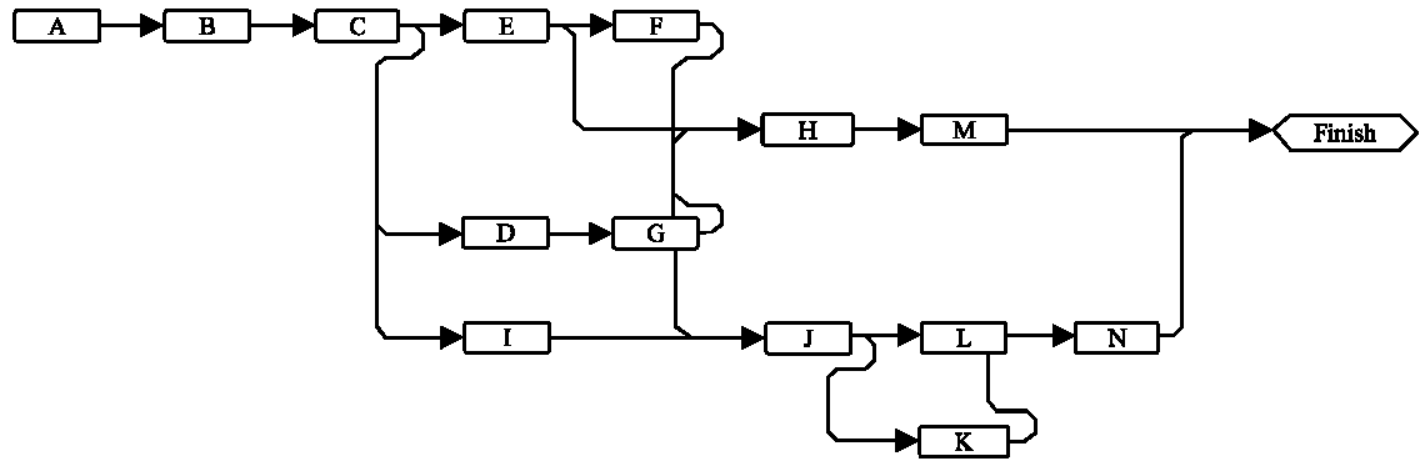

Fig. 2: Network diagram for case study in Table 1

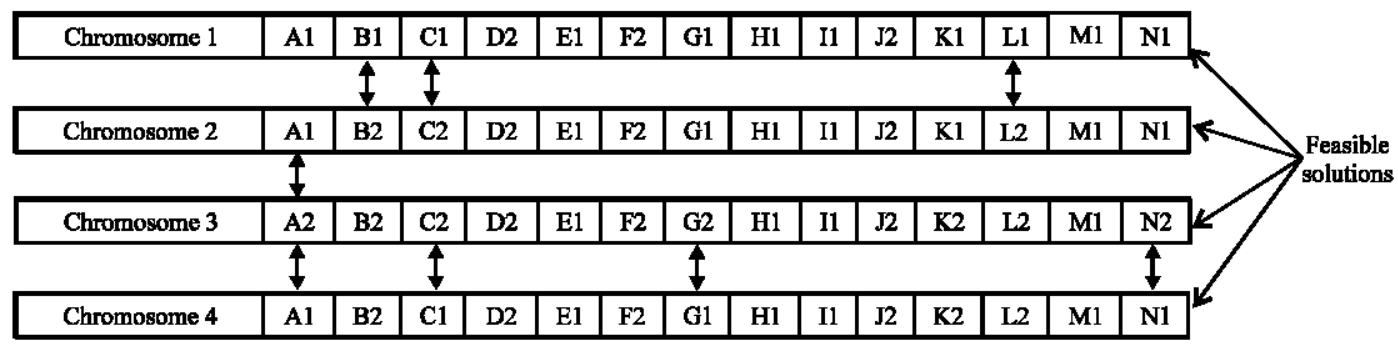

Fig. 3: The poject activity options as genes within the chromosomes and point up the process of changing genes between chromosomes 


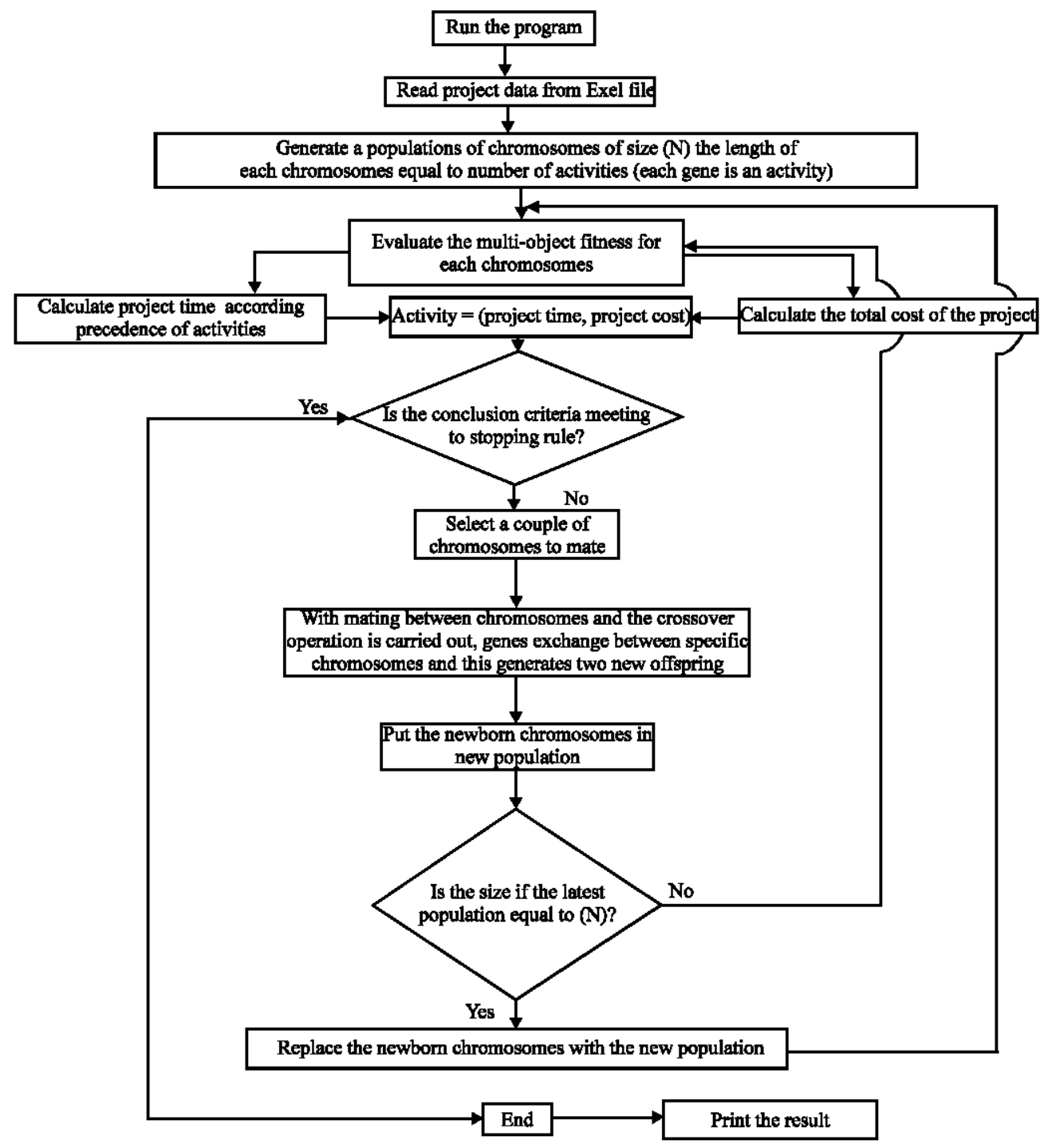

Fig. 4: A flow chart for the implementation MOGA procedure

\section{RESULTS AND DISCUSSION}

Figure 5 shows optimal solution set that generated by GA in scatter diagram, the doted curve augury to Pareto front, the stars in the graph represent the optimal solutions of Pareto front. Pareto front shows the trade-off between the objective 1 (Project time) and objective 2 (project cost) which shows that reducing project time increases its cost.

The minimum value (26 weeks) on the $\mathrm{X}$-axis represents the time of the project when employing the options of the method that takes the least time foreach activity and this corresponds to the highest cost on the $\mathrm{Y}$-axis $(\$ 62000000)$ the maximum value (44 weeks) on the $\mathrm{X}$-axis represents the time of the project when

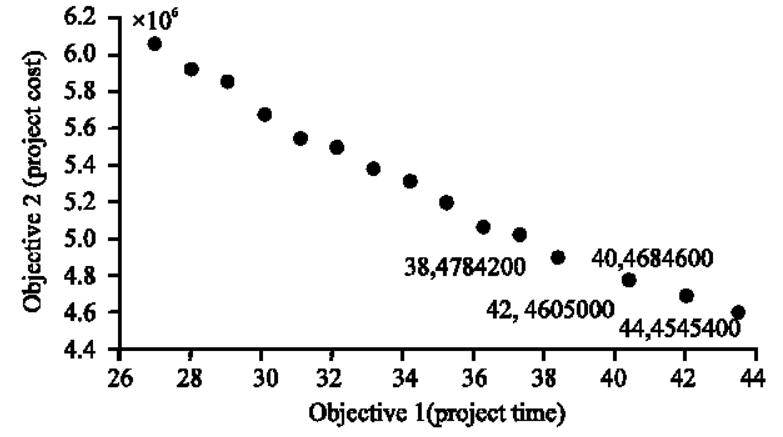

Fig. 5: The trade-off between time and cost, depending on the pareto front curve. The stars that appear in the drawing are listed on the Pareto front which represents the list of optimal solutions 
Table 2: Optimal solutions for trade-off between project time and its cost with selected options for activities

\begin{tabular}{|c|c|c|c|c|c|c|c|c|c|c|c|c|c|c|c|c|}
\hline \multicolumn{5}{|c|}{ Solution No. } & \multicolumn{10}{|c|}{ - Selected methods for each } & \multirow{2}{*}{$\frac{\text { Project time (weeks) }}{42}$} & \multirow{2}{*}{$\frac{\text { Project } \operatorname{cost}(\$)}{4,605,000}$} \\
\hline 1 & A1 & B1 & $\mathrm{C} 1$ & D2 & E1 & F1 & G1 & H1 & I1 & $\mathrm{J} 2$ & K1 & L1 & M1 & N1 & & \\
\hline 2 & A1 & B1 & $\mathrm{C} 1$ & D2 & E1 & F1 & G1 & H1 & I1 & $\mathrm{J} 1$ & K1 & L1 & M1 & N1 & 44 & $4,545,400$ \\
\hline 3 & A1 & B1 & $\mathrm{C} 1$ & D2 & E1 & F2 & G1 & H1 & I1 & $\mathrm{J} 2$ & K1 & L1 & M1 & N1 & 40 & $4,684,600$ \\
\hline 4 & A2 & B2 & $\mathrm{C} 2$ & D2 & E1 & F2 & G1 & H1 & I1 & $\mathrm{J} 2$ & K2 & L2 & M1 & N1 & 32 & $5,262,800$ \\
\hline 5 & A1 & B2 & $\mathrm{C} 1$ & D2 & E1 & F2 & G1 & H1 & I1 & $\mathrm{J} 2$ & K1 & L1 & M1 & N1 & 38 & $4,784,200$ \\
\hline 6 & A1 & B2 & $\mathrm{C} 2$ & D2 & E1 & $\mathrm{F} 2$ & G1 & H1 & I1 & $\mathrm{J} 2$ & K2 & L2 & M1 & N1 & 33 & $5,163,000$ \\
\hline 7 & A1 & B2 & $\mathrm{C} 3$ & D2 & E1 & F2 & G1 & H1 & I1 & $\mathrm{J} 2$ & K2 & L2 & M1 & N1 & 31 & $5,302,600$ \\
\hline 8 & A1 & B2 & $\mathrm{C} 2$ & D2 & E1 & F2 & G1 & H1 & I1 & $\mathrm{J} 2$ & K1 & L2 & M1 & N1 & 34 & $5,123,200$ \\
\hline 9 & A2 & B2 & C3 & D2 & E1 & F2 & G2 & H1 & I1 & $\mathrm{J} 2$ & K2 & L2 & M1 & N2 & 27 & $5,701,200$ \\
\hline 10 & A2 & B2 & $\mathrm{C} 3$ & D2 & E1 & F2 & G1 & H1 & I1 & $\mathrm{J} 2$ & K2 & L2 & M1 & N1 & 30 & $5,402,400$ \\
\hline 11 & A2 & B2 & $\mathrm{C} 2$ & D2 & E1 & F2 & G2 & H1 & I1 & $\mathrm{J} 2$ & K2 & L2 & M1 & N2 & 29 & $5,561,600$ \\
\hline 12 & A2 & B2 & $\mathrm{C} 1$ & D2 & E1 & F2 & G1 & H1 & I1 & $\mathrm{J} 2$ & K2 & L2 & M1 & N1 & 35 & $5,023,400$ \\
\hline 13 & A1 & B2 & C3 & D2 & E1 & $\mathrm{F} 2$ & $\mathrm{G} 2$ & H1 & I1 & $\mathrm{J} 2$ & K2 & L2 & M1 & N2 & 28 & $5,601,400$ \\
\hline 14 & A1 & B2 & $\mathrm{C} 1$ & D2 & E1 & $\mathrm{F} 2$ & G1 & $\mathrm{H} 1$ & I1 & $\mathrm{J} 2$ & K1 & L2 & M1 & N1 & 37 & $4,883,800$ \\
\hline 15 & A1 & B2 & $\mathrm{C} 1$ & D2 & E1 & $\mathrm{F} 2$ & G1 & H1 & I1 & $\mathrm{J} 2$ & K2 & L2 & M1 & N1 & 36 & $4,923,600$ \\
\hline 16 & A2 & B2 & $\mathrm{C} 3$ & D2 & $\mathrm{E} 2$ & $\mathrm{~F} 2$ & $\mathrm{G} 2$ & $\mathrm{H} 2$ & I2 & $\mathrm{J} 2$ & $\mathrm{~K} 2$ & L2 & M1 & N2 & 26 & $6,100,000$ \\
\hline
\end{tabular}

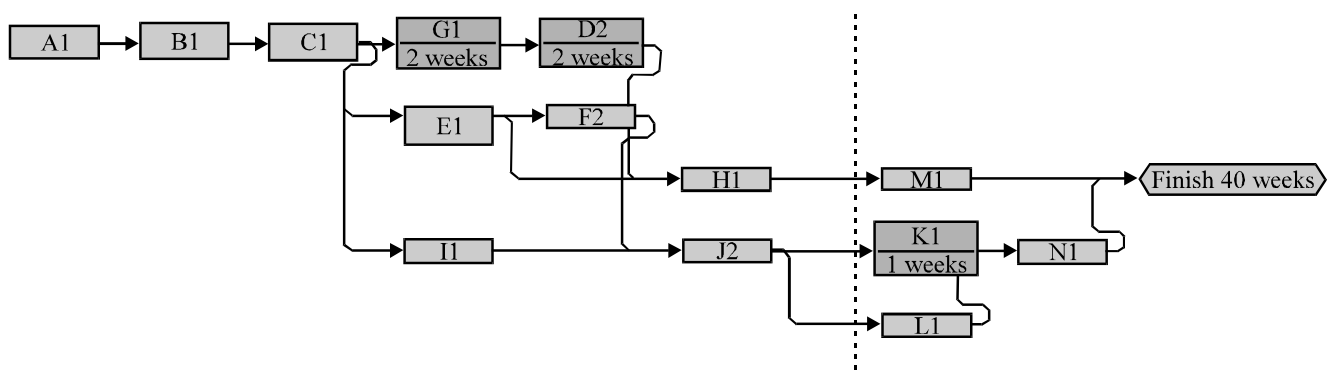

Fig. 6: Network diagram for solution No. 3

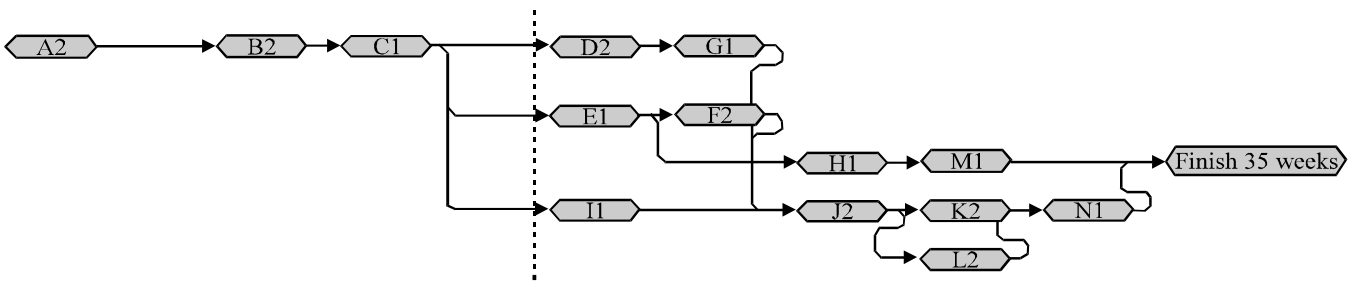

Fig. 7: Network diagram for solution No. 12

employing the options of the method that takes the maximum time for each activity at cost $(\$ 45454000)$.

Table 2 presentes 16 optimal solutions generated by GA based on Pareto front, displays the trade-off between time and cost as well as the method to be selected for each activity that provides the optimal solution set. And shows that change the method of work of any activity changes the time and cost to accomplish the project as a whole, for example, solution No. 3 shows that, the project to be completed by 44 weeks and $\$ 4,545,400$, activities must be completed with the method (A1 B1 C1 D2 E1 F1 G1 H1 I1 J1 K1 L1 M1 N1). By changing the method of activity $(\mathrm{J})$ and (f) to the second option, the project will be completed with estimation time of 40 weeks and cost $\$ 4,684,600$.

Depending on the trade-off between the time and cost of the project shown in the Pareto front curve and Table 2, the decision-maker can make the appropriate decision to satisfy it. In the case of management interest to complete the project at minimum possible time its can choose the solution No. 16 which assumes that the project ends with 26 weeks and methods of work for 14 activty in this solution are (A2-B2-C3-D2-E2-F2G2-H2-I2-J2-K2-L2-M1-N1) but its cost is very high (\$6100000).

In the case that the management has a budget dedicated to the performance of the project $\$ 4,684,600$ they can choose solution 3 which assumes that the project ends with 40 weeks and the options for working methods and running activities in this selection are (A1-B1-C1-D2-E1-F2-G1-H1-I1-J2-K1-L1-M1-N1).

To identify the critical activities and the amount of float time for non-critical activities of the project for solution 3 , the selected activity options are set in the MS project program which illustrates the network diagram as shown in Fig. 6 and 7. Pink activities are critical activities. 
Blue activities are non-critical activities that show the float time. In case a solution was selected No. 12, the network of critical activities as it is in the research network in Fig. 7. The network in Fig. 7 shows that all activities are critical. In order to complete the project by 35 weeks, no delay should be given to any activity otherwise, the completion of the project will be delayed.

\section{CONCLUSION}

The algorithm does not give a single optimal solution but a number of optimal solutions that the decision maker can choose the best of them for him. The proposed algorithm can deal with more objectives in the future (such as quality, environment, etc.,) by simply using the new objective equation and introducing it to the algorithm program.

The algorithm can handle projects with a very large number of activities more than 1000 activities and find a list of optimal solutions quickly with high efficiency. The algorithm helps the project management to choose the appropriate time based on the budget allocated for the completion of the project, this facilitates decision making for the decision-maker to know all the possible times to finish the project and to know its corresponding costs. And find out the best options for the work of the project activities for each optimal solution.

The results proved the effectiveness and efficiency of the algorithm in trade-off between the time of the project and its total cost with Pareto front. The proposed algorithm is useful and profitable for companies and project management where they qualify to choose the best methods to perform project activities from a list of optimal solutions.

\section{REFERENCES}

Afruzi, E.N., E. Roghanian, A.A. Najafi and M. Mazinani, 2013. A multi-mode resource-constrained discrete time-cost tradeoff problem solving using an adjusted fuzzy dominance genetic algorithm. Sci. Iranica, 20: 931-944.

Aminbakhsh, S. and R. Sonmez, 2016. Pareto front particle swarm optimizer for discrete time-cost trade-off problem. J. Comput. Civ. Eng., 31: 04016040-104016040-10.
Bajpai, P. and M. Kumar, 2010. Genetic algorithm-an approach to solve global optimization problems. Indian J. Comput. Sci. Eng., 1: 199-206.

Ding, C.H., S. Fujimura, X. Wei and W. Wei, 2010. Timecost trade-off problem under uncertainty incorporating multi-objective genetic algorithm and fuzzy sets theory. Proceedings of the $2010 \mathrm{IEEE} 17 \mathrm{th}$ International Conference on Industrial Engineering and Engineering Management, October 29-31, 2010, IEEE, Xiamen, China, ISBN:978-1-4244-6483-8, pp: 290-294.

Hooshyar, B., A. Tahmani and M. Shenasa, 2008. A genetic algorithm to time-cost trade-cost trade off in project scheduling. Proceedings of the 2008 IEEE International Congress on Evolutionary Computation (IEEE World Congress on Computational Intelligence), June 1-6, 2008, IEEE, Hong Kong, China, ISBN:978-1-4244-1822-0, pp: 3081-3086.

Ke, H., W. Ma and Y. Ni, 2009. Optimization models and a GA-based algorithm for stochastic time-cost tradeoff problem. Applied Math. Comput., 215: 308313.

Konak, A., D.W. Coit and A.E. Smith, 2006. Multi-objective optimization using genetic algorithms: A tutorial. Reliab. Eng. Syst. Saf., 91: 992-1007.

Mazlum, M. and A.F. Guneri, 2015. CPM, PERT and project management with fuzzy logic technique and implementation on a business. Procedia Soc. Behav. Sci., 210: 348-357.

Paryzad, B. and N.S. Pour, 2013. Time-cost-quality trade-off in project with using invasive weed optimization algorithm. J. Basic Appl. Sci. Res., 3: 134-142.

Sahu, K. and M. Sahu, 2014. Cost and time and also minimum project duration using alternative method. Intl. Rev. Appl. Eng. Res., 4: 403-412.

Van Sickel, J.H., P. Venkatesh and K.Y. Lee, 2008. Analysis of the pareto front of a multi-objective optimization problem for a fossil fuel power plant. Proceedings of the 2008 IEEE Power and Energy Society General Meeting On Conversio and Delivery of Electrical Energy in the 21 st Century, July 20 24, 2008, IEEE, Pittsburgh, Pennsylvania, ISBN:978-1-4244-1905-0, pp: 1-8. 